\title{
An overview of Design and Analysis techniques of Bootlace Lens for Multiple beamforming
}

\author{
G.S. Tomar, MIEEE, \& Ravi Kushwah
}

\begin{abstract}
A review of the design and analysis techniques reported so far for Bootlace type lens and its origin the Rotman type lens has been presented. The basic design and the improvement with its implications and adoptions are presented with the justification. The basic bootlace lens with the modifications reported with improvement and their characteristics is also presented with mathematical approach. The parameters and methods, which are of importance in the design and analysis of the work, are compared and performance thereon is considered for practical approach and fabrication results of some of the tested designs are also investigated. The basic design of microwave lens is Rotman lens and modifications have brought the new concept of bootlace lens due to its specific design and look as of open lenses of military boot. This analysis has given us direction for new approach in design.
\end{abstract}

Index Terms - Antenna array, Bootlace lens, Path length error, Rotman lens.

\section{INTRODUCTION}

In radar and advanced communication applications, it is necessary to scan a wide area for efficient communication. The Parabolic reflector based antenna is most commonly used for this purpose. The setup with parabolic reflector is shown in figure no. 1, which was most popular and easy to fabricate. However, this arrangement has major disadvantages when put into use; they are:
a. Difficult to realize large aperture due to mechanical constraints like back up structure and gears.
b. Blockage by the feed structure.
c. Slow in response as related to scanning in comparison to electronics scanning. Suffers reliability problem from shock and vibration.
d. Due to edging, angle of coupling and other mechanical disorders are common defects.
e. Difficult to align and requirement of alignment frequently.

To overcome these problems encounter in this arrangement an antenna array can be an alternate to

Manuscript received March 9, 2009. This work was partly supported by Dept of Science and Technology, Govt. of India and Defence Research and Development Organization New Delhi India for sponsored projects from respective departments.

First author is with the Vikrant Institute of Technology and Management, Indore, 452009 India as director of the Institute. Phone +91-9425129523 Fax: +91-7324-224005;

Second author is with Madhav Institute of Technology and Science, Gwalior 474005 India as research scholar in the department of electronics engg., parabolic reflector based antenna system, which is shown in figure no. 2 .
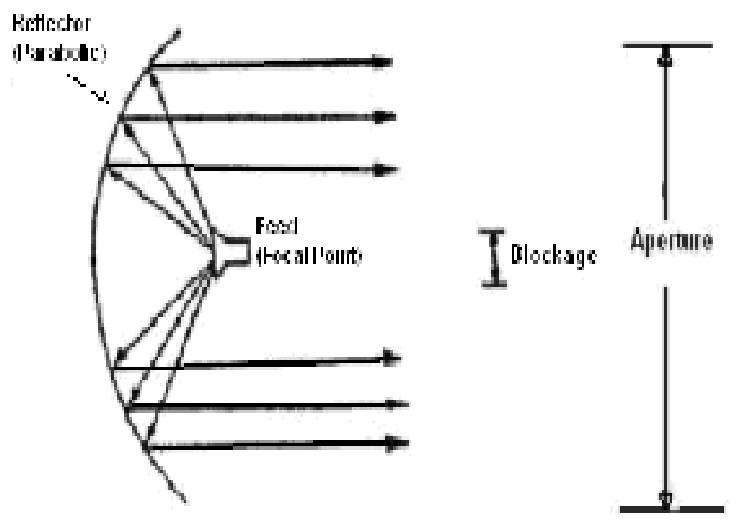

Figure 1 : Parabolic reflector antenna

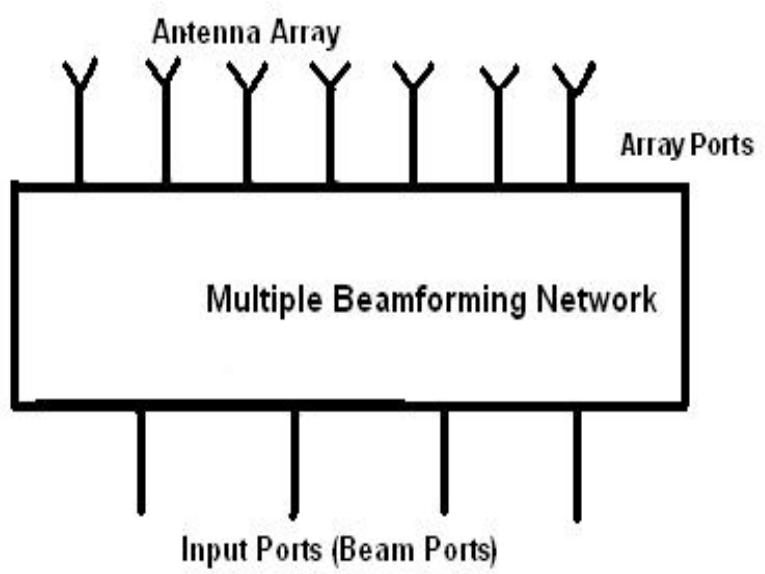

Fig. No. 2: Antenna Array with Multi Beam Network

A multiple beam-forming network is used to control the amplitude and phase at each element of the antenna array. Two types of multiple beam forming networks are available:

(i) Circuit type multiple beam forming network.

(ii) Microwave Rotman/ bootlace lens.

The circuit type multiple beam forming network [1] (as shown in figure 3 ) consisting number of directional coupler, phase shifter, power divider, cross-over network etc. The circuit type multiple beam forming networks are very complex especially the phase shifters are costly to fabricate and introduce considerable RF losses. This design could be of 
helpful for less number of input/ output ports.

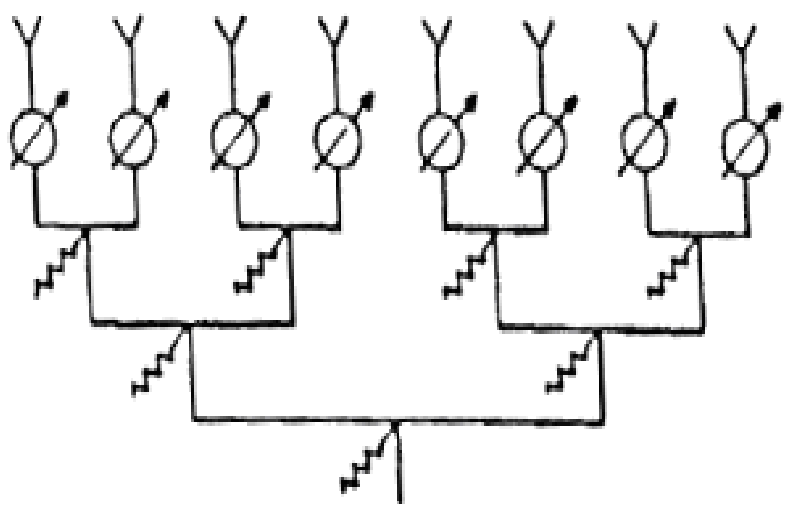

Figure 3 - Circuit Type Multiple Beam Forming Network

For large number of input-output ports this design becomes extremely complicated. Microwave bootlace lens suggested by Rotman and Turner [2] is a useful alternative to this complicated circuit type multiple beam forming network. This design was further corrected by D. Larry Leonakis [3]. This modification gave boost to the practical fabrication and testability of the lens.

\section{LENS GEOMETRY AND DESIGN APPROACH}

The microwave beam forming lens is conceptually operating in optical principle though it does not have any direct relation with its working. The cross section of tri-focal Rotman/ Bootlace type lens is shown in figure 4.

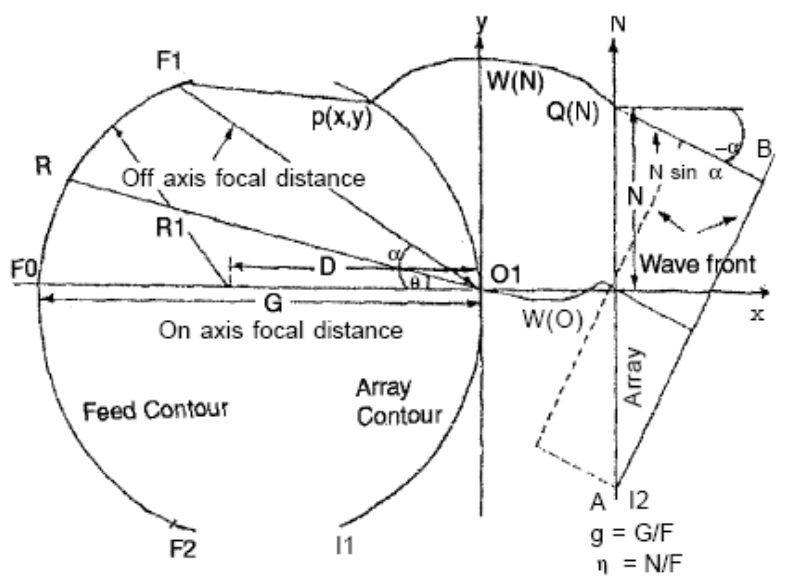

Figure 4 - Trifocal Rotman type Lens

In this design, one focal point $\mathrm{F}_{0}$ is located on the central axis and two other focal points $F_{1}$ and $F_{2}$ are symmetrically located on either side on a circular focal arc. Contour $\mathrm{I}_{2}$ is a straight line and defines the position of the radiating elements. $\mathrm{I}_{1}$ is the inner contour of the lens and also called the array contour. The inner and outer contours are connected by TEM mode transmission line $\mathrm{W}(\mathrm{N})$. Two off axis focal points $F_{1}$ and $F_{2}$ are located on the focal arc and make angels $\alpha$ and $-\alpha$ with the $\mathrm{X}$-axis. It is required that the lens be designed in such a way that the outgoing beam make angles $-\alpha, 0$ and $\alpha$ with the $\mathrm{X}$-axis when feeds are placed at $\mathrm{F}_{1}, \mathrm{~F}_{0}$ and $F_{2}$, respectively. Figure 4 shows the direction of outgoing beams for input at different feed ports. Rotman lens has the following four design parameters:

(i) Off axis focal length F: This is distance between off-axis focal point and midpoint of the array contour (this is distance $\mathrm{O}_{1} \mathrm{~F}_{1}$ or $\mathrm{O}_{1} \mathrm{~F}_{2}$ as defined in figure 4).

(ii) On axis focal length G: This is the distance between on axis focal point $\mathrm{F}_{0}$ and mid point of the array contour (this is distance $\mathrm{O}_{1} \mathrm{~F}_{0}$ as defined in figure 4).

(iii) Antenna element spacing d: Radiating elements are located along the straight line $\mathrm{I}_{2}$. Number of antenna elements and spacing between antenna elements determines the length of contour $\mathrm{I}_{2}$, also called lens aperture.

(iv) Focal angle $\boldsymbol{\alpha}$ : Focal angle determines the angular coverage provided by the lens. The lens can cover an angular area $\pm \alpha$. The angular coverage is dependant of its size and shape.

A ray originating from $F_{1}$ may reach the wave-front through a general point $\mathrm{P}(\mathrm{X}, \mathrm{Y})$ on the inner contour $\mathrm{I}_{1}$, transmission line $\mathrm{W}(\mathrm{N})$ and point $\mathrm{Q}(\mathrm{N})$ on the outer contour and then tracing a straight line at an angle $-\alpha$, terminate perpendicular to the wave-front $\mathrm{AB}$ at an angle $-\alpha$ to the broad side. Also, the ray from $\mathrm{F} 1$ may reach the wave front from $\mathrm{F}_{1}$ to point $\mathrm{O}_{1}$ and then through transmission line $\mathrm{W}(\mathrm{O})$, terminate at the wave front AB. Similarly; rays from other feed points may reach their respective wave front. Inner contour $I_{1}$ and the transmission lines are designed from the design equations which are derived using the fact that at the wave front all these rays must be in phase, independent of the path they travel. This required that the total phase shift in traversing the path to reach the wave front in each case be equal. The lens design equations can be written by equating the path length from the focal points to the corresponding wave front $[2,3]$.

The wide angle scanning capabilities of the bootlace lens are described in Archer [4,5], these types of lens are wide band systems. Since design is based on the different optical path length comparison. However, their bandwidth is dependent on the circuit elements used for the realization of the lenses such as: array elements, connecting TEM transmission lines, the array geometries and mutual coupling effects. The bandwidth is defined taking various points of views and summarized by Frank [6]. He built a ninety-six element-scanning array which operates over an octave. The array beam is steered with digital latched/switched ferrite phase shifters, which have low insertion loss and constant phase shift over entire octave. The dielectric transformers were used for having perfect matching between input and radiating apertures. This has been tested for $60^{\circ}$ scanning angle but on higher frequency range some beams are lost over $60^{\circ}$ scanning angle.

\section{OTHER GEOMETRIES AND DESIGN OF LENS}

The conventional design approach of Rotman lens [1,2] is based on ray optics tracing technique. It suggested that the lens could be designed by optical path length comparison 
such that the path length from the focal point to any point on the corresponding wave front is constant.

Shelton [7] suggested modification in the design and stated that it is not necessary to have circular focal arc and a lens with front-to-back symmetry was designed. In this design input ports and array ports can be interchanged. The reasons for choosing symmetrical configuration are:

(a) an asymmetrical lens will have either the lens arc or the feed arc more strongly curved than the arcs obtained for the symmetric solution. The more curved arc will have larger port spacing and a larger variation in port spacing from the centre to the edge.

(b)Symmetric lens exhibits better aberration characteristics.

(c) The tendency for feed ports to illuminate other feed ports is more severe for asymmetric lens.

(d) The symmetric lens is smaller than an equivalent asymmetric lens.

Katagi et al [8,9] suggested an improved design method of Rotman lens in which a new design variable is introduced and the phase error on the aperture of the antenna array is minimized. In this design the exciting elements and receiving elements are arranged on a curve. Three focal points are taken for feed points and the aperture length of a linear array antenna is twice of max N. it is also suggested that the shape is not necessarily a circular arc as it is in conventional design [1] and there is freedom in design of lens.

David R. Gagnan [10] presented an alternative approach to design the lens by refocusing the focal arc according to the Snell's law and termed the new lens as refracting lens. It was designed in microstrip configuration. This approach provides beam ports and array ports placements, which gives improved coupling to the outermost beam ports, particularly for strip line or microstrip configuration used with small array antennas.

Y.M. Tao et al [11] designed the printed circuit lens fed multiple beam antenna array for millimeter wave indoor personal communication systems, which is formed by a linear array of equally spaced elements fed with an improved printed circuit Rotman lens. Both the lens and the array are designed and built on the same dielectric substrate. The antenna operates in the frequency range of $27-30 \mathrm{GHz}$ and covers an angular sector near to $120^{\circ}$ with 11 beams. Using this design the size of lens is further minimized. The lens was implemented with wave-guide technology to minimize losses in the millimeter wave region. It consists of a parallel plate region with wave-guide ports distributed around the periphery of the plates. Beam forming or focal ports are located on one side of the plate. These ports are fed by a switch array. The array ports are on the opposite side, each connected to $\mathrm{n}$ antenna element. Energy input into a specific focal port will emerge from the antenna elements and produce a beam along a particular direction

KK Chan et al [12] designed a Rotman lens feed network for a hexagonal array of oversized radiating elements. The performance of a hexagonal array of radiating elements fed by a rectangular lattice of identical row and column boards of Rotman lens is described. The resultant beams with high directivity and good isolation are arranged in a hexagonal lattice that is rotated $90^{\circ}$ from the element lattice. To reduce the size of the lens, dummy ports on the array/beam port contour must be eliminated so that the array/beam ports are contiguous. For this purpose, elements spacing is smaller and does not permit grating lobes.

$\mathrm{K}$ Inagaki et al [13] have developed a beam-forming network for three independent steerable beams by a five element array antenna integrated on a birefringent photonic integrated circuit (PIC) chip. Phase shift characteristics of BFN are measured by optical wavelength tuning. In a coherent optical signal processing system transmitting an RF signal as the frequency difference of two light waves, the phase of the regenerated RF signal from the light waves is controlled by changing the relative phase difference between the two light waves, which required only a small optical path control on the order of micrometers. The operating principle of the BGN is based on the combination of an optical Rotman lens technique for multibeam information and an optical wavelength scanning technique to steer each beam independently. A star coupler, delivering input beam signal to output element signals acts as the optical Rotman lens forming a fixed multi beam. The beam-forming network consists of two ports.

KK Chan et al [14] presented a simple and compact network scheme for feeding hexagonal shape planar array to produce multiple beams. Linear multiple beam forming networks are directly connected row-wise and then column-wise in an egg-crate fashion to feed the array. All the linear networks are identical which is advantageous for low-cost implementation. The resultant beams are distributed in U-V space on a triangular grid that is rotated $90^{\circ}$ from that of the element grid. Performance and characteristics of such an array architecture using a wide band Rotman lens network to feed a horn array. Feeding a planar array with a passive network to produce multiple beams is straight forward if the beam, element and network lattice are all rectangular. The array elements are typically fed row wise and then column wise using linear multiple beam forming network.

J.J. Lee et al [15] have proposed RF heterodyne technique for Rotman lens to reduce the size of beam forming network. This design was proposed for airborne antenna operating in L-band of frequency range. The Rotman lens with 48 output ports and 60 input ports is chosen as base line approach for proposed design. The Rotman lens can be designed to have a single focal point or multiple focal points, which depends on area to be scanned, level of side lobes and phase arrays. This design is an alternate design for highly dielectric loaded Rotman lens to reduce the size. The highly loaded dielectric Rotman lens suffers with drawbacks of heavy weight and greater ohmic loss.

E.O. Rausch et al [16] have developed a millimeter wave Rotman lens that operates at frequencies between $33 \mathrm{GHz}$ and $37 \mathrm{GHz}$ with the potential of being implemented in moralized plastic. It is electronically scanned millimeter wave Rotman lens design. The various lens design were evaluated with a computer model passed on the contour integral method. The best lens design was manufactured and characterized by measuring the scattering parameters between the beam array ports within the frequency interval of interest. In this the focal points are located on one side of the plate and fed by switch array. The array ports are on the 
opposite side connected to antenna elements. The input energy given for a specific focal port will merge from antenna element and produce a beam in particular direction. The input from focal port to focal port is switched electronically in one direction within the plane of the lens. The designed lens has 32 active antenna elements port, 17 active beam ports and the maximum scan angle of $\pm 22.2^{\circ}$. The maximum scan angle upto $\pm 60^{\circ}$ and frequency range upto $95 \mathrm{GHz}$ are possible with different lens designs. The measured parameters are compared with computed and found $-30 \mathrm{~dB}$ maximum sidelobe level and less than $2.3 \mathrm{~dB}$ insertion loss.

M.C.D. Maddocks et al [17] have designed a flat plate steerable antenna for satellite communication and broadcast reception. He has given two implementation of the Rotman lens: ( i ) use a multiport lens and (ii) Parallel plate lens with a single movable beam port.

The G/T of the antenna system is also improved by reducing loss in the Rotman lens and feed network. The side lobe level is reduced by improved control of mismatches in the design and tolerance in the assembly.

R.C. Hansen [18] has designed Rotman lens by using six basic design parameters; focal angle, focal ratio, beam angle to ray angle ratio, maximum beam angle, focal length and array element spacing. The last two are in wavelengths, and beam angle to ray angle ratio of sins. The seventh design parameter allows the beam port arc to be elliptical instead of circular.

He also described the effects of the seven design parameters on the shape and the geometric phase and amplitude of Rotman lens. The lens shape is important, both in conserving space and reducing loss. Wide lenses have large spillover loss and higher transmission line loss. A compact lens tends to minimize spillover losses; roughly equal; ports curve heights now become important, to avoid severe asymmetric amplitude tapers and large phase errors.

D.L. Johnson et al [19] designed an offset pillbox reflector with a $2^{\circ}$ maximum $\mathrm{H}$-plane $-3 \mathrm{~dB}$ beam width and $-35 \mathrm{~dB}$ maximum side lobes over a $6 \mathrm{GHz}$ to $12 \mathrm{GHz}$ band. The linear array of 23 elements is primary feed for offset reflector. The desired amplitude distribution was for 79 inch pillbox antenna. The exit aperture was taken as sixth order polynomial distribution with $32 \mathrm{~dB}$ taper at the reflector edges and it was capable of producing $-45 \mathrm{~dB}$ side lobes.

L. Musa et al [20] have designed a microstrip Rotman lens port. In this design a microstrip port is viewed as a 2-D aperture antenna. An open ended waveguide radiator can be used having a cut angle end for beam deflection to a position between the aperture normal and the direction of the waveguide sides. In this design a cut angle microstrip port is assumed and might produce a deflection from the aperture normal to the direction defined by the guide sides.

This design of microstrip Rotman lens is for four beams from seven element array having eleven beam ports. The double use of ports in this design has $3 \mathrm{~dB}$ loss in an overlap network of hybrid junction in which it is used to create beams with low sided lobes and high cross-over.

H.L. Southall et al [21] have designed a completely overlapped sub array antenna for broad band. It has low side lobes, wide angle performance. The input is fed to input steering lens through phase shifter. This is transform feed which is Rotman lens and the objective is cylindrical bootlace lens. It has parallel plate wave guide cavities with coaxial probe elements. This design has $20 \%$ band width at $55^{\circ}$ scan and side lobe of $-30 \mathrm{~dB}$.

C.Metz et al [22] have designed millimeter wave monolithic integrated circuit technology of low profile antennas with high resolution capability as well as wide angular coverage in radar systems. In this design MMIC production technology is used to have $77 \mathrm{GHz}$ communication antenna.

M.J. Gans et al [23] have designed a dual polarized narrow beam antenna to use for geostationary arc coverage of $60^{\circ}$. The beam width is less than $0.5^{\circ}$.it produces conically scanned beam by means of linear array of feed horns with bais cut apertures illuminating a pair of parabolic cylinder reflector in a special arrangement of images. This design is relatively simple because it uses reduced size array and singly curved reflector. This has been checked for $0.35^{\circ}$ beam with in $\mathrm{Ku}$ band earth station having up to $0.05^{\circ}$ pointing accuracy and scan loss less than $1 \mathrm{~dB}$ for entire scan region.

K.K. Chan et al [24] have designed Rotman lenses which are used in ESM system for driving high power sources having wide azimuth coverage with multiple beams. The input ports arc and output port arc of this lens are synthesized based on the geometric optics principle. This method was especially developed for planar lens. The ports may be open circuited or connected to external feed lenses. The ports of the lens which are not connected to the input and output loads are terminated in to loads.

V.V. Denisenko et al [25 ] have designed an antenna which was used in automotive intelligent cruise control applications. This design consist two separate printed circuit antennas having one transmit antenna of wide beam width and three narrow beam width receiving antennas. The Rotman lens multibeam forming network is used for providing multi beam operation in receiving antennas. Directivity of this arrangement is $36 \mathrm{~dB}$ and $31 \mathrm{~dB}$ for receive and transmit antennas which have around $6 \mathrm{~dB}$ losses. This antenna system has very high isolation but higher side lobes.

P. Phu et al [26] have developed a wide bandwidth electronic scanning antenna (ESA) which is based on a Rotman lens beam forming network with shared aperture. It allows multiple beams to transmit simultaneously in different directions and at different frequencies. This design supports high bandwidth form 8 to $18 \mathrm{GHz}$ frequency band.

R.A. Sparks et al [27] have designed and developed a fiber optic Rotman lens using silica glass planar waveguide couplers and suitable lengths of single mode optical fiber. The beam former used in this design was form factored photonic integrated with a wideband linear antenna array for providing eight beams pointing in the directions corresponding to $\pm 7.5^{\circ}, \pm 22.5^{\circ}, \pm 37.50$ and $\pm 52.5^{\circ}$. This design was used for radar and communication systems for producing multiple beams simultaneously.

C. Metz et al [28] have designed fully integrated multi chip module and an electronically steerable microstrip patch array for automotive application in radar sensor. The antenna feed system used in this design is modified Rotman lens. This has provided an extended angular coverage having better resolution and can was used for automatic cruise control at 77 GHz. In this design no moving part is used hence it is rugged 
and durable with high reliability. In this design by using cut in excitation at the array port and using power splitter to the feeding network, broad beam is generated for more area coverage and having precrash sensing capability.

K.K. Chan et al [29] have designed a rectangular lattice with identical row and column boards of Rotman lenses for feeding hexagonal array of radiating elements. The resultant beams are arranged in a hexagonal lattice that is rotated $90^{\circ}$ from the element lattice. This design has good isolation and high directivity. It is capable of generating multiple spot beams arranged in a hexagonal lattice form a planar array where the hexagonal element lattice is rotated $90^{\circ}$ relative to beam lattice.

T. Katagi et al [30] proposed an improved design method of Rotman lens antenna. In this design method the phase error on the aperture of linear array is minimized and especially for large array length the phase error is very small. This design is basically for small and low loss Rotman lens antenna.

Rappaport et al [31] designed three dimensional bifocal and quadrufocal bootlace lenses using curved focal arc. Due to availability of curved focal arc for three dimensional bifocal and quadrufocal bootlace lenses. This design is difficult but reliable, compact and having low insertion loss. In this design the scan performance is drastically improved and phase aberration performance of the lens across the field is improved by locating the feed elements on an optimum surface. In quadrufocal lens phase aberration is less than the bi-focal lens scan.

J.S. Herd et. al. [32] have designed and fabricated of an eight microstrip antenna array feed by a microstrip Rotman lens. The method of moment was used to find the feed point optimization is more accurate. Microstrip Rotman lens is fabricated for broad band multiple beam feed. It has been found that feed point optimization by means of a method of moments solution has highly accurate, and that the microstrip Roman lens was an inexpensive fabricated broadband multiple beam feed structure.

K.D. Claborn et al [33] have given the computer aided design of an electronically scanned Rotman lens. The FORTRAN language is used for writing the computer program. To derive the geometry of the lens by using the BPORT program gives the satisfactory result by varying the parameter of the lens. The far field pattern of the lens was computed with different excitation of input probe.

\section{ANALYSIS APPPROACHES OF BOOTLACE LENS}

A conventional ray optics design of multiple beam forming networks gives information about the phase distribution at antenna array elements. It does not give information about the amplitude distribution at the array elements and radiation pattern of the array elements.

Katagi et al [8,9] analyzed Rotman lens for path length error obtained by the improved method. For large array length, the phase error on the aperture due to this improved method becomes considerable smaller than due to conventional method. Using this improved design there is greater freedom in designing Rotman lens.
Smith et al [34-35] developed a theoretical model using 2-D aperture theory to predict the amplitude performance across array ports and insertion loss of the lens. This model treats the lens beam ports and array ports as 2-dimensional antenna. The aperture theory is used for the flared waveguide case and would also be suitable for a microstrip lens. To calculate power received by each array port Friis 2-D transmission formula is derived. Insertion loss can be calculated by using these results. The amplitude distribution is used with the phase calculated from the geometrical path length approach to predict the radiation patterns of the lens fed array at various frequencies. It was established that the experimental results are near to theoretical predictions with some basic differences.

P.C. Sharma et al [36] reported an alternative two dimensional field analysis approach for analysis of Rotman lens. Z-matrix of the lens is computed for its multiport model using contour integration method. Z-matrix was used to evaluate $S$ parameters. A radiation pattern for the antenna array is computed using the field distribution. The analysis results and experimental results were forum tin agreement.

David R. Gagnon [37] have derived a procedure for proper refocusing of the dielectric filled Rotman lens with beam port locations determined according to Snell's law. This approach resulted in an alternative lens configuration, which may give a wider field of scan at a given focal length for lenses fabricated in microstrip or stripline configuration.

V.K. Tripp et al [38] have given the dispersion characteristics of a Rotman lens. The transfer functions of the lens measured by the broadband characteristics of the lens. The transfer function has multiply by the spectrum of input pulses to get the complete frequency response. The time domain response containing the distortion was obtained by fast Fourier transform method.

P.S. Hall et al [39] have presented the review of the radio frequency antenna beam forming techniques for scanned and multiple beam antennas. The multiple beam antenna has the capability to produce many beams in different direction from the same aperture. The multi beam antenna does not require any device to change the frequency. The hybrid reflectors and lenses will produce high gain beam over narrow scan ranges. Circuit beam formers have traveling waves or corporate feed characteristics and can be used in limited size array to give wide bandwidth.

A.A. Shishegar et al [40] have proposed a hybrid analysis method for planar lens type structure. In this $\mathrm{N}$ number of input waveguides are connected to the input surface of a planar lens at different positions and $\mathrm{N}$ number of output waveguides are connected to the output surface. The lens like medium between the two surfaces is large in terms of wavelength. The transmission characteristics of this structure are analyzed by using asymptotic technique.

N. Yuan et al [41] have given the hybrid procedure that combines the least square finite element method and transfinite element method for analysis of multiport rotman lenses. The LSFEM is employed to built a function based on the residuals of the full first order Maxwell equation governing the electromagnetic fields in the structure. As the full first order Maxwell equation method is free form spurious solutions. The transfinite method is used to transfer the exterior conditions in the port region to the interior 
region. This method is applied to a rotman lens to determine the field distribution in the lens and the scattering parameters Rotman lens when implemented in stripline or microstrip becomes a planar multiport network. The rotman lens used for comparison consists of nine input ports, eight output ports and sixteen dummy ports, which are terminated into matched $50 \Omega$ loads.

J. T. Loane et al [42] have given the various constrained lenses or reflector equation with relation to the abbe sine condition. The optical path within such lenses is generally constrained to follow a waveguides or other transmission line.

J. Paul Shelton [43] has given the focusing characteristics of symmetrically configured bootlace lenses. The design lens was no limitation on shape of the feed curve and the lens has the front to back symmetry. The geometric optics ray tracing technique was used for the analysis of lens. The symmetric lens exhibit better aberration characteristics and the symmetric lens are smaller than asymmetric lens. The lens has been design in such a way to produce $\pm 90^{\circ}$ coverage from a $\lambda / 2$ spaced array of radiators. They also calculate the lens dimensions and focusing characteristics. The maximum usable array length in the range of $20 \lambda$ to $200 \lambda$, the ratio of lens width to array length varies from 1.0 to 1.6 and wave front tolerance is $\lambda / 16$. This type of lens is called unscaled because the scale factor relating the lens size and the radiating array size is chosen arbitrarily.

K.K.Chan et al [44] have designed a broad band and wide scan angle. Rotman lens. They used geometric optics principle for the analysis of input port and output port with assumption that antenna element spacing are fixed. The variables are used for designing the lens are off-axis focal point beam angle.

S.G. Kim et al [45] have presented a electronically controlled antenna for wireless communication base station. This type of antenna consists of microwave switch, a symmetrical bootlace lens like. Rotman lens and linearly arrayed antenna. The. Rotman lens is used here to feed a linear array antenna to produce multiple beams.

By using a microwave lens and microwave switch, a new phased array base station antenna s proposed with electronic and remote controllable so that the traffic can not be taken off or re-routed whenever the down tilt change is desired also the maintenance and installation cost is further reduced.

Andrew F. Peterson et al [46] have given the numerical techniques for the analysis of micro strip, strip line and waveguide Rotman lenses.

The lens was fabricated in stripline, microstrip and waveguide at frequency range of $2 \mathrm{GHz}$ to $40 \mathrm{GHz}$. The $\mathrm{S}$-parameter is obtained for comparison with numerical models. For measuring the beam pattern, side lobe levels, mutual coupling between feed, shape and composition of lens sidewall numerical formulation was used.

W.F. Richards et al [47] have given an efficient technique for computing the potential Green's function for a thin, periodically excited parallel plate waveguide bounded by elctric and magnetic walls. The Green's function is used for evaluating the cavity potential. The Green's function is computed over a wide band of frequencies with little additional effort required for a single frequency.
M.J. Maybell et al [48] have designed a broadband Rotman lens array. Pattern synthesis is used by exciting three or more ports of a. Rotman lens fed array. Choosing beam spacing and beam amplitudes to sample the desired pattern at the six beam peak angles synthesize the radiation pattern. The side lobes are analyzed at $11.5 \mathrm{GHz}$ center frequency where it is found nearly $-35 \mathrm{~dB}$ at $0.4^{\circ}$ beam width.

M.J. Maybell et al [49] have also given another analysis model by using ray structure method for coupling coefficient. It has been analyzed for two-dimensional Rotman lens. The mutual coupling and reflection coefficient was analyzed by using this ray structure method.

A. Tomasic et al [50] have given the performance of a coaxially fed monopole element in an infinite linear phased array radiating into a semi-infinite parallel plate guide. By the knowledge of mutual coupling, amplitude and phase of transmitting and receiving signal a Rotman lens of low side lobe level was analyzed.

P.K.Singhal et al [51] have analyzed various Rotman lens design using contour integral approach. It was analyzed by dividing feed contour and array contour. The dummy ports were terminated by $50-\Omega$ load.

\section{CONCLUSION}

The bootlace lens is an attractive alternate to the other multiple beam forming networks. By using new invention in the field of microwave engineering, the compact size of antennas and beam forming network is possible. By looking to the various designs considered in this work, the size of this network and antenna has come to the stage where antennas of higher gain may not be visible outside the communication equipment and can be operated from few $\mathrm{GHz}$ frequency range to $100 \mathrm{GHz}$ frequency range with high efficiency and reliability. Now in the age of global communication this may be very helpful in reducing size increasing traffic rate and efficiency by adopting the new approaches presented so far. The application of this lens is also proposed for modern communication systems like adhoc network and wireless communication as suggested by some design approaches.

\section{REFERENCES}

[1] Butler J. And Lowe R., " Beam forming matrix simplifies design of electronically scanned antennas", Electronics design, 9 pp.170-173, 1961.

[2] W. Rotman and R.F. Turner, "wide angle microwave lens for line source applications", IEEE Trans. On Antennas \& Propagat .,Vol.Ap-11, pp 623-632,Nov.1963.

[3] G. Larry Leonakis, "correction to wide angle microwave lens for line source applications”, IEEE Trans. on Antennas \& Propag., Vol.36, No.8, pp 1067Aug.1986.

[4] D. Archer, "Lens Fed multiple-beam arrays", Microwave J.Vol.18, pp 37-42,1975.

[5] D. Archer, "Lens Fed multiple-beam arrays", Microwave J. Vol.18, pp 171-195, Sept. 1984

[6] J. Frank, "Bandwidth criterion for phased array antennas", Proceedings of phase array antenna symposium, Artech House, Dedham M.A.1972.

[7] J. P. Shelton, "Focusing characteristics of symmetrically configured bootlace lenses", IEEE Trans. on Antennas \& Propag. Vol.AP-26, pp 513-518, July 1978.

[8] T. Katagi, S. Mano and Shin-Lchi sato " An improved design method of Rotman lens Antennas" , IEEE.Trans on Antennas \& Propag.Vol.Ap-32, pp 524-527, may 1984 . 
[9] T. Katagi, S.Mano S .Sato, P.K. Singhal and P.C .Sharma, "Comment on correction to an improved design method of Rotman lens Antennas" IEEE Trans. On Antennas \& Propaga,Vol.43, No.6, June1995,p634.

[10] D.R. Gagnon, "Procedure for correct refocusing of the Rotman lens according to Snell's law", IEEE Trans. On Antennas Prop. Vol. 37, No.3, pp390-392, March 1989.

[11] Y.M. Tao and G.Y. Delisle "Lens-Fed Multiple Beam array for millimeter wave indoor communications", A\&P Society International Symposium , 1997.IEEE.,1997 Digest Volume 4, 13-18 July 1997.

[12] K.K. Chan and S.K. Rao "A Rotman lens feed Network for a hexagonal Array of Oversized Radiating Elements", A\&P Society International Symposium ,2000. IEEE, Vol.1 16-21 July 2000.

[13] Keizo Inagaki, Weiwei Hu, Yoshihiko Mizuguchi and Takashi Ohira "Birefringent Photonic Integrated circuit for beam forming network of independently steerable multibeam antenna", Laser and Electro-Optic Society 2000 Annual Meeting.Leos $200013^{\text {th }}$ Annual meeting IEEE Volume: 1, 13-16 Nov 2000.

[14] K.K. Chan, "Planar waveguide model of Rotman lenses", IEEE ApSymp. Dig .pp 651-654, 1989.

[15] J.J.Lee, G.W.Valantine "Multibeam array using Rotman lens and RF Heterodyne" A\&P Society International Symposium, AP-S. Digest, Volume: $321-23$ july 1996.

[16] E.O.Rausch, A.F.Peterson and W. Wiebach " electronically scanned millimeter wave antenna using a Rotman lens" Radar 97. 14-16 Oct.1997.

[17] .C.D.Maddocks and M.S.Smith, "A flat plat Steer-able antenna design for satellite Communication and broadcast reception" Antenna and Propagation, 1989, ICAP 89 Sixth International Conference on 4-7 Apr 1989.

[18] R.C.Hansen, "Design trades for rotmann lenses", IEEE Trans. On Antennas \& Propagat. Vol. 39, No.4, pp 464-472, April 1991

[19] Deron L.Johnson, Michael J.Maybell and Joseph A.Troychak "Octave Bandwidth,- 35dB Sidelobe Single Offset Pillbox Reflector Using A Rotman Lens and Array as a Primary feed" A\&P Society Internationa Symposium ,1987, Volume: 25, Jun 1987

[20] L. Musa and M.S Smith, "Micro strip Port design and side wall absorption for printed Rotman lenses" Microwave Antenna and propagation ,IEE proceeding H. Volume:136 Issue :1 ,Feb.1989.

[21] Hugh L. Southall and Daniel T.McGrawth, “ An Experimental Completely Overlapped Subarray Antenna"Antennas And Propagation. IEEE Transaction on Volume:34 Issue: 4 April 1986

[22] Carsten Metz, Jens Grubert, Johann Heyen, A.F.Jacob, S.Janot. "Fully Integrated Automotive Radar Sensor With Versatile Resolution" Microwave Symposium Digest,2001 IEEE MTT-S International , Volume: 2, pp 1115-1118, 20-25 May 2001

[23] Michel.J.Gans and Noach Amitay "Narrow Multibeam Satellite ground Station Antenna Employing a Linear Array with a Geosynchronous Arc Coverage of 60 degree" 1983

[24] K.K.chan "Planar Waveguide Model of Rotman lens" IEEE Trans on CH-2654.2 /89, 1989.AP-S , digest, vol 2., pages :651-654 26-30 June 1989

[25] Vladimir V. Denis enko, Anatoly G. Shublov, Alexender V. Majorov, "Millimeter-wave Printed Circuit Antenna system for Automotive Application" Microwave Symposium Digest, 2001 IEEE MTT-S international, Volume : 3, pp 2247 -2250 20-25 May 2001

[26] Phuong Phu, and James Lilly "A Wide Band Width Electronic Scanning Antenna for Multimode RF Sensing” IEEE Trans on 07803-4977, pp 177-180, 20-22 April

[27] R.A.Spark and N.Slawsby and J.Prince and J.Munro, “ Eight Beam Prototype Fiber Optic Rotman Lens" Microwave Photonics, 1999.MWP '99. International Topical Meeting pp 283-286, vol.1, 17-19 Nov.1999

[28] Casten Metz, Jens Grubert, Johann Heyen, “Fully Integrated Automotive Radar Sensor with Versatile Resolution" Microwave Theory and Techniques, IEEE Transaction on volume: 49, 12 Dec . 2001

[29] Kwok Kee Chan and Sudhakar Rao, "Design of a Rotman Lens feed Network to Generate a Hexagonal Lattice of Multiple Beam", IEEE Transaction on Antenna and Propagation ,Volume :50, Issue :8 ,pp 1099-1108,Aug.2002

[30] Takashi Katagi and Seiji Mano, "An Improved Design Method of Rotman Lens Antennas" IEEE Transaction on Antenna and Propagation, Vol:32, Issue:5 pp 524-527, May 1984.

[31] C.M. Rappaport and A.I. Zaghloul, "Optimized three dimensional lenses for wide angle scanning", IEEE Tans. On Antenna and propagat, Vol. Ap.33, No.11,pp 1227-1236, Nov. 1985

[32] Jeffrey S.Herd and David M.Pozar " Design of A Micro strip Antenna Array Fed By Arotman Lens" A\&P Society International Symposium,1984,Volume:22, pp 729-732, Jun 1984

[33] K.D.Claborn, J.A.Gailant , and R.E.Willey, A.I.Sinsky, "Computer Aided Design of an Electronically Scanned Rotman Lens" Antenna and propagation Society International Sumposium ,1977,Volume:15, pp 353-356, June 1977

[34] M.S. Smith and A. K.S. Fong, " Amplitude performance of Ruze and Rotman lenses", The Radio and Electronic Engineer ,Vol.53, pp329-336,Sept.1983

[35] A.K.S. Fong and M.S. Smith, "A micro strip multiple beam forming lens" The radio and Electronic Engineer,Vol.54,NO.7/8, pp318-320,1984

[36] P.C. Sharma, K.C. Gupta, C.M.Tsai, J.D.Brice and R. Presnell, "Two-dimensional field analysis for CAD of Rotman type beam forming lenses", International J. of microwave and Millimeter-Wave Computer Aided Engg. Vol.2,No.2,pp 82- 89, April 1992

[37] David R Gagnon, "Procedure for correct refocusing of the Rotman lens according to Snells law". Antennas and Propagation, IEEE Transaction on, Volume :37 Issue:3 March 1989

[38] Victor K.Tripp, John E.Tehan and Carl W.White "Characterization of the Dispersion of a Rotman lens" A\&P Society International Symposium,1989. AP-S. Digest, 26-30 June 1989

[39] P.S.Hall and S.J.Vetteriein, "Review of Radio Frequency beam forming techniques for scanned and multiple beam Antenna", Microwaves Antenna and Propagation, IEE Proceeding H Volume :137, Issue :5, Oct 1990.

[40] A.A. Shishegar; S. Safavi-Naeini, “ A hybrid analysis method for planar lens-like structuires", Antennas and propagation society International Symposium, AP-S Digest, Vol. 3, pp 2012-2015, 21-26 July 1996.

[41] N.Yuan, J.S.Kot and A.J.Parfitt "Analysis of Rotman lenses using a hybrid least Squares FEM/ transfinite element method "Microwaves, Antennas and Propagation IEE Proceeding -.Vol 148, Issue: 3, pp 193-198, June 2001

[42] Joseph T.Loane and David R Tanner " General Constrained lens or Reflector Equations with Relations to the Abbe Sine condition." A\&P Society International Symposium, 1996, P-S Digest volume: 2, pp 926-927, 21-26 July 1996.

[43] J. Shelton "Focusing Characteristics of Symmetrically Configured Bootlace lenses" IEEE Transaction on Antennas and Propagation,Volume:26 ,Issue:4 pp 513-518, July 1978

[44] K.K. Chan and W. Tam, "Field analysis of planar bootlace lens feeds", Int., Conf. on Radar Paris France, Vol. I, pp 273-278, April24-28,1989.

[45] S.G.Kim, S.M.Park, C.w.ro and J.m.Lee, " Electronic Beam Tilting of Base Station Antenna Rotman Lens Fed" radio and Wireless Conference, 1998,RAW Con 98. 1998 IEEE,9-12 Aug, pp 95-98, 1998

[46] Andrew F. Peterson and Ekkehart O.Rausch, " Scatting Matrix Integral Equation Analysis for the Design of a Wave guide Rotman Lens", Antennas and propagation, IEEE transactions on ,Volume :47, Issue : 5, pp 870-878, may 1999

[47] William F.Richards and Kim Mcinturff and Peter S. Simon, "An Efficient Technique for Computing the Potential Green's Functions for a Thin, Periodically Excited Parallel-plate Waveguide Bounded by Electric and Magnetic Walls" Microwave Theory and techniques, IEEE transactions on, Volume:35, Issue :3, pp 276-281, may 1987

[48] Michel J.Maybell, “ Printed Rotman Lens-Fed Array having Wide Bandwidth, Low Side lobes, Constant Beam width and Synthesized Radiation Pattern" Antennas and Propagation Society International Symposium ,1982,volume:20, pp136-139 may 1982

[49] Michel J.Maybell "Ray Structure Method for Coupling Coefficient Analysis of the Two Dimensional Rotman Lens", Antennas and Propagation Society International Symposium, 1981,Volume: 19, pp 144 -147 , Jun 1981

[50] A.Tomasic and A.Reseal " Linear Phased Array of Coaxially-Fed Monopole Elements In a Parallel Plate Guide"Antennas and Propagation Society International Symposium, 1982,volume: 20, pp144-147, may 1982

[51] P.K.Singhal and R.D.Gupta with P.C.Sharma "Investigation of the Effect of Design Parameter on the Performance of Rotman Type Multiple Beam Forming Network" Proceedings of APMC 2001, pp 593-596, Jul 2001.

First Author completed undergraduate, postgraduate and doctoral degree in the field of electronics engineering. He has total work experience of more than 22 years in the field of practical applications with Indian defense and in academics. he is senior member of IACSIT and Member of IEEE. Presently he is working on applications of new design concepts of bootlace lens in mobile adhoc and cellular communication and also on acoustic imaging for new sponsored projects from government of India. 